Patient Knowledge and Attitudes Toward Cervical Cancer Screening after the 2012

\title{
Screening Guidelines
}

Jayanti M. CLAY, MD ${ }^{\mathrm{a}}$; Joanne K. DAGGY, PhD ${ }^{\mathrm{a}}$; Sunetris FLUELLEN, MD ${ }^{\mathrm{a}}$; Brownsyne TUCKER EDMONDS, MD, MPH, MS ${ }^{\mathrm{a}, \mathrm{b}}$

aDepartment of OB/GYN, Indiana University School of Medicine, Indianapolis, USA

${ }^{\mathrm{b} C o r r e s p o n d i n g ~ a u t h o r ~}$

Corresponding author at:

Address: $410 \mathrm{~W} 10^{\text {th }}$ Street, Indianapolis, IN 46202

Phone : (317) 944-1661_Email : btuckere@iupiu.edu

Highlights:

- Patients had limited awareness of cervical cancer and current screening practices.

- Patients expressed discomfort with deviating from annual screening.

- Most patients did not perceive themselves to be at risk for cervical cancer.

- Most lacked understanding of the components of cervical cancer screening.

- Cervical cancer screening knowledge significantly increased with education level.

\begin{abstract}
OBJECTIVE: To assess women's attitudes and preferences related to recent changes in cervical cancer screening guidelines. METHODS: We distributed 380 surveys in three University based and Community clinics. Study participants anonymously completed surveys, which included questions related to demographics, cervical cancer, screening practices, risk perception and
\end{abstract}

\section{CERVICAL CANCER SCREENING KNOWLEDGE AND ATTITUDES}


attitudes towards changing practices. RESULTS: 315 women agreed to participate (83\%). $60 \%$ $(185 / 310)$ of participants had some college education or higher and $12 \%(36 / 305)$ worked in the medical field. On average, participants answered $4.1(\mathrm{SD}=1.3)$ of the 8 knowledge questions correctly. Knowledge scores significantly increased with education level (Kruskal-Wallis test pvalue $<0.001)$. The majority $(72 \%, \mathrm{n}=228)$ reported that they should be screened annually, and that screening should be initiated with the onset of sexual activity $(63 \%, n=197)$. Participants that were more knowledgeable of current screening practices were more comfortable extending screening intervals (Kruskal-Wallis test $\mathrm{p}<0.001$ ). CONCLUSION: Even among a relatively highly educated population of women, participants had limited knowledge of cervical cancer and current screening guidelines. Many participants reported discomfort with less frequent screening intervals. PRACTICE IMPLICATIONS: This study supports the need for improvement in cervical cancer prevention education especially with regards to the new screening guidelines.

Keywords: cervical cancer; prevention; screening; guidelines; knowledge; attitudes

\section{INTRODUCTION}

Over the last forty years, Pap smear screening has enabled early detection and removal of precancerous and cancerous cervical lesions, resulting in a substantial decline in cervical cancer incidence and mortality in the United States (US) [1,2]. US cervical cancer screening guidelines have evolved since 2002 due to growing concerns about the harms of overtreatment of precancerous lesions, including: patient discomfort, anxiety, bleeding, inconvenience, increased health care costs, cervical insufficiency leading to preterm deliveries and perinatal mortalities [3]. In light of these concerns, as of 2012, the American Society for Colposcopy and Cervical Pathology (ASCCP) no longer recommends cervical cancer screening under the age of 21, and has lengthened the recommended interval for screening from annual screening to screening and 
co-testing of cervical cancer and HPV every three to five years for women ages 21-30 and 30-65, respectively [4].

A 2013 vignette-based survey of gynecology providers in Indiana revealed discordance between their cervical cancer screening practices and 2012 screening guidelines, with only $16.2 \%$ of providers completely adhering to the current guidelines [5]. A similar study in the Pacific Northwest noted that $48 \%$ of the provider participants were not following or planning to follow the 2012 guidelines [6]. Both these studies found that providers were concerned about meeting their patients' expectations to have annual or frequent screenings $(52.7 \%$ and $66.1 \%$, respectively) as reasons for not complying with the current standard of care [5,6]. Additionally, studies of factors influencing patients' attitudes and compliance with cervical cancer screening prior to the new guidelines include ethnicity, socioeconomic status, insurance status, age, education and profession [7-12]. Particularly, uninsured minority women of low socioeconomic status with low levels of education are less likely to be screened according to current guidelines [7-9].

It is important that patients become familiar with and understand the new screening guidelines so that providers are not faced with choosing between satisfying patient expectations and practicing evidence-based medicine. Failing to recognize and attend to this potential conflict may result in over-screening. For patients, this may lead to unnecessary procedures, patient morbidity and increased healthcare costs $[13,14]$. Unfortunately, there is limited data on patient knowledge of cervical cancer, current screening guidelines, patients' perceived risk of cervical cancer with declining incidence and attitudes towards these changing screening practices. Our primary objective is to understand patients' knowledge of cervical cancer and Pap tests; attitudes towards less frequent screening; and perception of risk to underscore areas for improvement in 
patient education. Our secondary objective is to understand the factors that are associated with higher knowledge levels, higher perceived risk and favorable attitudes towards the new guidelines.

\section{METHODS}

With approval from the Institutional Review Board of Indiana University (IRB \# 1309131062; approval date: 01/08/2014), under exempt status, we conducted a survey among a convenience sample of women recruited in three outpatient women's health clinic sites in Indianapolis associated with Indiana University Hospital, Health Net and Eskenazi Hospital systems. These sites incorporated a university based faculty clinic, a resident clinic with faculty supervision and a federally qualified community health center staffed by attending physicians. The survey was performed in English and Spanish. Inclusion criteria were female, age greater than 18 years, ability to comprehend English or Spanish, ability to consent and willingness to participate in the survey. Patients attending these clinics over an eight-week period were asked of their willingness to participate in the study. Those interested in participating were given an anonymous survey instrument to privately complete and envelopes to seal completed surveys. Surveys were returned at the same visit.

Following a detailed review of current literature on knowledge and attitudes towards cervical cancer and Pap tests and adapted previously published question stems and survey items, resulting in a 25-item survey developed to assess knowledge of cervical cancer and Pap smears, attitudes towards screening practices, risk perception as well as demographic information [8-10]. The knowledge portion of the questionnaire consisted of six multiple-choice items and two true or false items. The instrument was pre-tested with a small number of women of reproductive age to test reading level, understandability, and time to completion. 
Table 2 displays each item and response set. Each multiple-choice question had one correct answer and two to four distracters. Each item was scored as either correct or incorrect. The total number of correct responses provided the knowledge score, which could then range from 0 to 8 . There were four questions examining the participants' attitude towards Pap tests that included a free text field in addition to multiple-choice questions. Two of these questions evaluated their comfort level and response to increasing intervals between screenings. The other two questions evaluated their fear or discomfort with the test and their perception of the test's role in cervical cancer prevention. Study participants rated their responses on a 5-point Likert scale ranging from strongly agree to strongly disagree. Additionally, one question assessed risk perception of cervical cancer with a 5-point Likert scale as noted above. The remainder of the survey collected demographic and pertinent medical history information. The survey was pilot tested at the University site prior to distribution at other sites.

Descriptive statistics were calculated for all survey questions. The total number correct for the 8 items examining patient knowledge was calculated. In the knowledge scale, when at least $50 \%$ of the items in that scale were non-missing, we assumed an imputed mean for that item based on the mean of the available items for that person. For the $\mathrm{N}=315$ participants that completed surveys, only 9 participants completed part of the knowledge questions. Only one person completed fewer than $50 \%$ of the items thus was excluded from analysis. When examining associations of knowledge with patient demographics, missing demographic items were not imputed. Bivariate associations between patient knowledge and categorical demographic characteristics were assessed using either Wilcoxon-rank sum tests or KruskalWallis tests as the total number correct is not normally distributed. Spearman correlation was used to assess the association of total number correct with 
other continuous measures. Fisher's exact test was used to test bivariate associations between categorical variables. Missing data for survey questions was excluded from subgroup analysis. Statistical analysis was completed using SAS V9.4 (SAS Institute Inc., Cary, NC).

\section{RESULTS}

A total of 380 surveys were distributed at the different clinic locations of which 315 surveys $(83 \%)$ were completed and included in the analysis. Of the 10 surveys that were only partially completed, nine were still included in analysis regarding patient knowledge as less than $50 \%$ of knowledge items were missing.

Baseline characteristics are listed in Table 1. Ages ranged from 18 to 73 with approximately $\mathbf{8 0 \%}$ falling below age 40 . The ethnic representation was similar to that of Indianapolis with majority of the study participants being of Caucasian (56\%) followed by African American (29\%). Approximately, 12\% of the participants reported to work as pharmacists, registered nurses, medical assistants, medical students and physicians. Of the 33\% of participants under age 26 , there was a 45\% (39/86) vaccination rate for the HPV vaccine. Knowledge

Responses to the individual knowledge questions are displayed in Table 2. On average, the participants answered $51.8 \%$ of the eight knowledge items correct. The median total number of knowledge items correct was four (IQR =2)), with 59\% $(186 / 314)$ of the participants answering between one and four of the eight knowledge questions correctly. Fewer than half (47\%) of the patients identified a Pap test to be a test for cancer of the cervix. More than $30 \%$ reported that a Pap test was a pelvic exam with a speculum. Fifteen percent correctly identified that cervical cancer screening should be started at age 21 , with $61 \%$ of participants reporting that Pap test screening per the guidelines should begin when women are sexually active. Most 
women continued to believe that they should be screened for cervical cancer annually, regardless of risk, with $72 \%$ of the participants reporting that the test needs to be performed every year. Almost $60 \%$ of the participants reported that screening should be continued for their lifetime. More than $76 \%$ of the patients reported that they could get a Pap test at places besides their clinic including the Emergency Department and Urgent Visit Centers.

\section{Bivariate associations between patient knowledge and demographic characteristics}

Knowledge did not significantly differ in participants with a history of cervical dysplasia (categorized as "yes" vs. "no/I am not sure") (Wilcoxon rank sum test p-value =0.338). However, age correlated with knowledge $\left(\mathrm{r}_{\mathrm{s}}=-0.04, \mathrm{p}\right.$-value $\left.=0.49\right)$. Likewise, knowledge significantly increased with education level $\left(\mathrm{r}_{\mathrm{s}}=0.352\right.$, $\mathrm{p}$-value $\left.<0.001\right)$. And study participants in a medical occupation had significantly more knowledge (p-value $<0.001$ ). Attitudes

Overall, only $49 \%$ of participants reported feeling comfortable with the new guidelines while $47 \%$ of participants would not feel comfortable with going more than 1 year between screenings. Of those who would not feel comfortable with going more than 1 year between screenings, $71.4 \%$ reported that they would ask to be screened annually. Additionally, $21 \%$ of those who reported feeling comfortable with 3-5 year screenings also reported that they would ask to be screened annually. Attitude significantly varied by knowledge (Fisher's exact test pvalue $<0.0001$ ) as illustrated in Table 3 . Of patients that correctly answered the screening interval frequency question, $90 \%$ reported comfort with current screening practices. When patients incorrectly reported that screening should be conducted annually, 59\% reported that they would not be comfortable with the current guidelines. Almost $5 \%$ of patients reported that they would ask for a more detailed explanation from their provider or would solicit a second opinion 
or would change providers if asked to follow these new recommendations. Only $11 \%$ of patients reported discomfort with the Pap test and further elaborated that this was due to discomfort with the procedure Risk perceptions

Only 39\% (45/311) of the patients felt that they might be at risk for cervical cancer. Approximately $32 \%$ disagreed or strongly disagreed that they were at risk and $29 \%$ were undecided on their perceived risk of cervical cancer. Notably, $99 \%$ of patients reported having been sexually active, 24\% of the patients reported a history of an abnormal Pap and $23 \%$ reported tobacco use. Patient's risk perception for cervical cancer did not significantly differ between age groups $(\mathrm{p}$-value $=0.893)$. Patients who answered yes to having an abnormal Pap test perceived themselves to be at higher risk (Kruskal-Wallis test $p$-value $=0.005)$. Perceived risk was also significantly higher for those who smoked tobacco $(\mathrm{p}$-value $=0.011)$ but did not differ based on HPV vaccine status ( $\mathrm{p}$-value $=0.901$ ) based on Wilcoxon rank-sum tests.

\section{DISCUSSION AND CONCLUSION}

\subsection{Discussion}

This study set out to determine the current knowledge base of women in regards to cervical cancer and Pap tests as well as their attitudes towards the new guidelines and their risk perception given the declining rates of cervical cancer. Overall, the respondents demonstrated a limited awareness of cervical cancer and current screening practices. The vast majority (72\%) of study participants believed that they should receive annual screening, and the majority would feel uncomfortable even with a physician's recommendation for less frequent screening.

Similar to findings by Robinson et al., our study identifies important deficits in cervical cancer screening knowledge [15]. Furthermore, previous studies by Vernilli et al. and King et al. 
reported that patient expectations drove provider deviation from guideline adherence [5,6]. Our findings that patients expressed discomfort with deviating from annual screening and that screening should not be stopped past age 65 suggest that these concerns are valid. For low risk patients with normal prior screens, more frequent screening increases healthcare costs and morbidity $[13,16]$. However, it is interesting that patients who were more knowledgeable of current screening practices were also more comfortable with the current screening guidelines. Notably, we collected these data almost two years after the guideline changes had been in effect. It is difficult to say how widely disseminated the changes were among the general public. No large scale public health educational campaigns were undertaken, and our institution did not create an education campaign. We suspect that patients would largely have been receiving education about the guideline changes from their providers. Which, interestingly, could mean that their perspectives could reflect provider concerns as much as their own. This suggests a pivotal role for patient education in improving patient attitudes towards these new guidelines.

Similar to the findings of Fry et al. in 2010, our study suggests that women may lack awareness that screening tests are not being performed at the time of pelvic examination [17]. In our study, $30 \%$ of the respondents believed a Pap test to be a speculum exam and $76 \%$ of the respondents believed that they could get cervical cancer screening in the ED and urgent visit centers, in addition to their clinic. This is concerning as respondents might accidentally interpret speculum exams in the ED and urgent visit center as their annual screening. As $60 \%$ of cervical cancers occur in women who have never been screened or screened in the last five years, these misconceptions place women at a higher risk for cancer [2]. This identifies a critical gap in physician-patient communication that may point to the need for additional training to 
ensure that physicians clearly convey the elements and purpose of the exam they are performing.

Most of our study participants did not perceive themselves to be at risk for cervical cancer. Despite their risk perception of "low to no risk status," the participants felt strongly about annual screening. Our study participants seemed to understand the association between abnormal Pap tests, smoking and cervical cancer and appropriately classified themselves to be at higher risk. Furthermore, our study demonstrated that receiving the HPV vaccination did not alter risk perception in this population. There are limitations to the study that must be considered in interpreting our findings. As a convenience sample, obtained from and urban academic medical system, the generalizability of our findings may be limited. Moreover, though our participants were representative of the Indianapolis community in terms of ethnicity and age, our study population may not be a representative sample given that more than half of the participants had taken college level courses with 30\% having completed an Associates' or Bachelor's degree and $12 \%$ worked in some capacity within the healthcare field. However, such a bias would likely bias our findings toward overestimating the knowledge women have regarding screening guidelines, as we would expect this population to have higher than average health literacy. Though knowledge scores improved with an occupation in the medical field, higher levels of education in the study population may have confounded this finding. Though existing measures would have been optimal, the limited literature informing our study question precluded our ability to utilize validated items. In turn, we adapted previously published surveys and pilot tested items for use in an exploratory capacity. Lastly, health literacy is a key conceptual domain which we failed to assess with our survey instrument. The addition of a health literacy scale may have yielded important insights into modifiable predictors of screening knowledge, attitudes or risk perceptions. 
Conclusion

Based on our findings, women are most comfortable with annual screening compared to the new guidelines that suggest getting screened every 3-5 years, and lack basic understanding of the components of their annual pelvic exam, cervical cancer screening and Pap tests. Additionally, almost half of the participants reported discomfort with the new screening guidelines. Our study suggests that further efforts are needed to engage patients in discussions about cervical cancer, mainly focusing on the difference between a pelvic exam and a Pap test, education regarding the 2012 cervical cancer screening guidelines, and risk factors for cervical cancer.

\subsection{Practice Implications}

Addressing and alleviating women's misconceptions of the pelvic exam, cervical cancer screening, and Pap tests may help minimize morbidity and reduce healthcare costs. Increasing patients' awareness of the current screening guidelines and the benefits of lengthened interval screening, may help to bridge the gap between patient expectations and evidence-based practice.

\section{CONFLICT OF INTEREST: none.}

DISCLOSURES: The authors report no conflict of interest. The corresponding author confirms that all bylined authors fulfilled the conditions for authorship. This research did not receive any specific grant from funding agencies in the public, commercial, or not-for-profit sectors.

\section{CERVICAL CANCER SCREENING KNOWLEDGE AND ATTITUDES}


Presented at the 2015 American College of Obstetricians and Gynecologists Annual Clinical and Scientific Meeting, San Francisco, CA, May 2-6, 2015

\section{REFERENCES}

[1] U.S. Cancer Statistics Working Group, United States Cancer Statistics: 1999-2010 Incidence and Mortality Web-based Report. https://www.cdc.gov/uscs, 2013 (accessed 4 Oct 2016).

[2] A. Jemal, R. Siegel, J. Xu, E. Ward, Cancer statistics, 2010, CA. Cancer J. Clin. 60 (2010) 277-300.

[3] D. Saslow, C.D. Runowicz, D. Solomon, A.B. Moscicki, R.A. Smith, H.J. Eyre, et al., American Cancer Society guideline for the early detection of cervical neoplasia and cancer, CA. Cancer J. Clin. 52 (2002) 342-62.

[4] D. Saslow, D. Solomon, H.W. Lawson, M. Killackey, S.L. Kulasingam, J. Cain, et al., American Cancer Society, American Society for Colposcopy and Cervical Pathology, and American Society for Clinical Pathology screening guidelines for the prevention and early detection of cervical cancer, CA. Cancer J. Clin. 62 (2012) 147-72.

[5] N.R. King, K.M. Kasper, J.K. Daggy, B. Tucker Edmonds, Current practice patterns in cervical cancer screening in Indiana, Am. J. Obstet. Gynecol. 210 (2014) 265 e1-8.

[6] L. Verrilli, R.L. Winer, C. Mao, Adherence to cervical cancer screening guidelines by gynecologists in the Pacific Northwest, J. Low. Genit. Tract Dis. 18 (2014) 228-34.

[7] K. Limmer, G. LoBiondo-Wood, J. Dains, Predictors of cervical cancer screening adherence in the United States: a systematic review, J. Adv. Pract. Oncol. 5 (2014) 31-41. 
[8] E.A. Borrayo, J.J. Thomas, C. Lawsin, Cervical cancer screening among Latinas: the importance of referral and participation in parallel cancer screening behaviors, Women Health. 39 (2004) 13-29.

[9] M. Bazargan, S.H. Bazargan, M. Farooq, R.S. Baker, Correlates of cervical cancer screening among underserved Hispanic and African-American women, Prev. Med. 39(2004) 465-73.

[10] N. Cook, E. Kobetz, I. Reis, L. Fleming, D. Loer-Martin, S.A. Amofah, Role of patient race/ethnicity, insurance and age on Pap smear compliance across ten community health centers in Florida, Ethn. Dis. 20(2010) 321-6.

[11] E.D. Paskett, J.M. McLaughlin, P.L. Reiter, A.M. Lehman, D.A. Rhoda, M.L. Katz, et al., Psychosocial predictors of adherence to risk-appropriate cervical cancer screening guidelines: a cross sectional study of women in Ohio Appalachia participating in the Community Awareness Resources and Education (CARE) project, Prev. Med. 50 (2010) 7480.

[12] J.K. Tracy, A.D. Lydecker, L. Ireland, Barriers to cervical cancer screening among lesbians, J. Womens Health. 19 (2010) 229-37.

[13] M. Arbyn, M. Kyrgiou, C. Simoens, A.O. Raifu, G. Koliopoulos, P. Martin-Hirsch, et al., Perinatal mortality and other severe adverse pregnancy outcomes associated with treatment of cervical intraepithelial neoplasia: meta-analysis, Brit Med J. 337 (2008) a1284.

[14] K.B. Roland, V.B. Benard, A. Soman, N. Breen, D. Kepka, M. Saraiya, Cervical cancer screening among young adult women in the United States, Cancer Epidemiol. Biomarkers Prev. 22 (2013) 580-8. 
[15] K. Robison, L. Clark, W. Eng, L. Wu, C. Raker, M. Clark, et al., Cervical cancer prevention: Asian-American women's knowledge and participation in screening practices, Womens Health Issues. 24 (2014) e231-6.

[16] ACOG Committee on Practice Bulletins-Gynecology, ACOG Practice Bulletin no. 109: Cervical cytology screening, Obstet. Gynecol. 114 (2009) 1409-20.

[17] A.M. Fry, E.A. Ferries-Rowe, L.A. Learman, D.M. Haas, Pap smear versus speculum examination: can we teach providers to educate patients?, J. Womens Health. 19 (2010) $1715-9$. 
Table 1. Baseline Characteristics of Study Participants $(\mathbf{N}=315)$

\begin{tabular}{|c|c|}
\hline Characteristic & $\mathbf{N}(\%)$ \\
\hline Total & 315 \\
\hline Age, mean \pm SD & $33 \pm 12$ \\
\hline \multicolumn{2}{|l|}{ Race } \\
\hline White & $174(55.9)$ \\
\hline Black & $90(28.9)$ \\
\hline Hispanic & $30(9.6)$ \\
\hline Asian & $6(1.9)$ \\
\hline Other & $11(3.5)$ \\
\hline \multicolumn{2}{|l|}{ Language } \\
\hline English & $280(90.6)$ \\
\hline Spanish & $22(7.1)$ \\
\hline Chinese & $1(0.3)$ \\
\hline Other & $6(1.9)$ \\
\hline \multicolumn{2}{|l|}{ Education } \\
\hline Less than HS & $29(9.4)$ \\
\hline HS diploma/GED & $96(31.0)$ \\
\hline Some college & $87(28.1)$ \\
\hline Associates degree & $21(6.8)$ \\
\hline Bachelor's degree or higher & $77(24.8)$ \\
\hline
\end{tabular}




\begin{tabular}{|l|c|}
\hline \multicolumn{2}{|l|}{ Occupation } \\
\hline Medical & $36(11.8)$ \\
\hline Other & $269(88.2)$ \\
\hline Insured & $307(99.4)$ \\
\hline Sexual activity (ever had sex) & $71(23.1)$ \\
\hline Tobacco Use & $23(7.4)$ \\
\hline Hysterectomy & $74(23.9)$ \\
\hline Abnormal Pap & $72(23.2)$ \\
\hline HPV Vaccine & $10(3.2)$ \\
\hline Immunocompromised & \\
\hline
\end{tabular}


Table 2. Responses to Knowledge Questions (Questions 1-3, 6-9, 13 on survey)

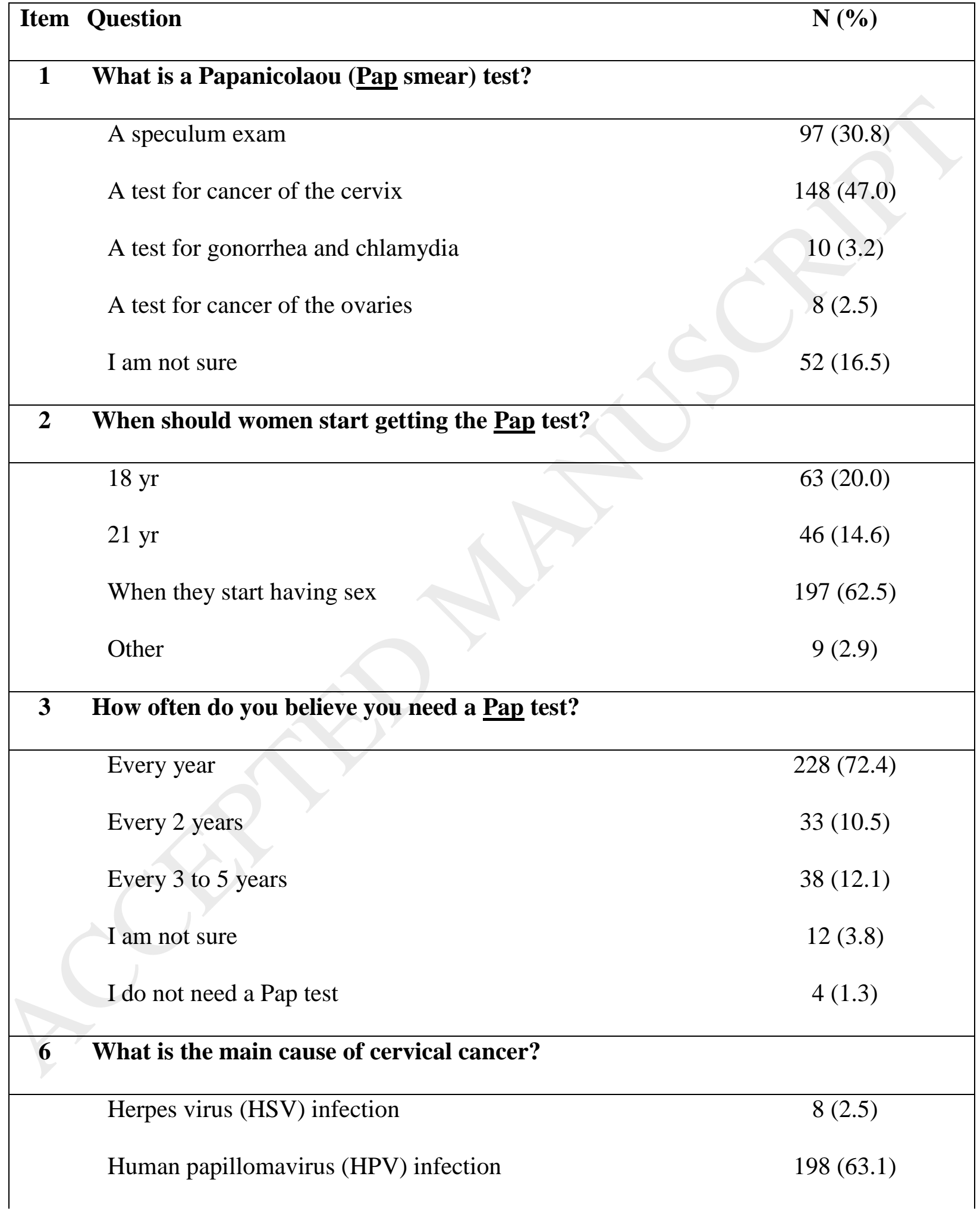




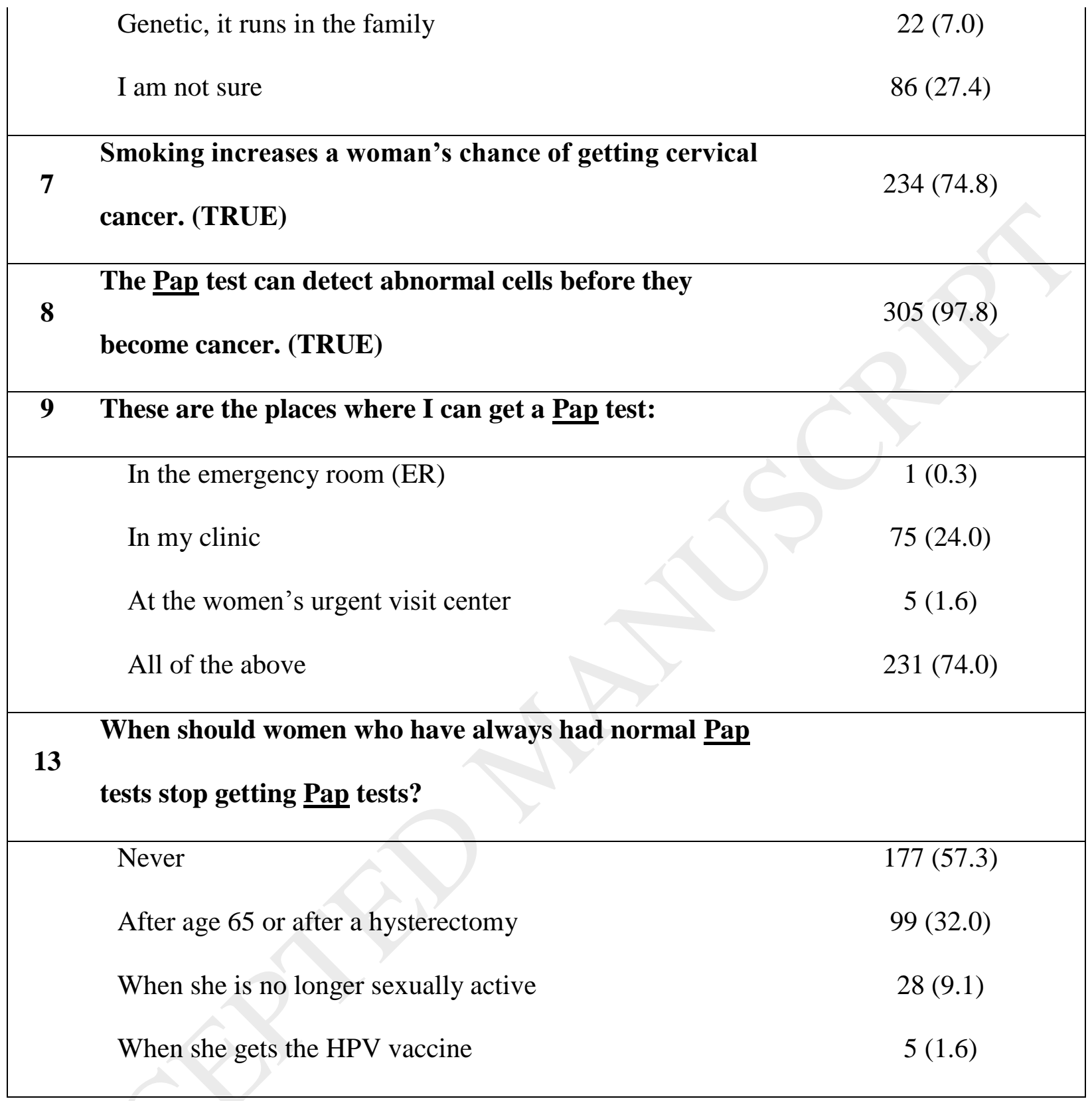


Table 3. Association of Knowledge of Current Screening Guidelines with Patient's Comfort Level

\begin{tabular}{|lccc|}
\hline & How comfortable would you be if your doctor told you that \\
& you only needed a Pap test every 3 years? \\
\hline How often do you believe & Comfortable & Not comfortable & Fisher's exact \\
you need a Pap test? & $\mathrm{N}(\%)$ & $\mathrm{N}(\%)$ & p-value \\
\hline Every year & $90(41.5 \%)$ & $127(58.5 \%)$ & $<0.0001$ \\
Every 2 years & $22(68.8 \%)$ & $10(31.3 \%)$ & \\
Every 3 to 5 years & $34(89.5 \%)$ & $4(10.5 \%)$ & \\
I am not sure & $8(72.7 \%)$ & $3(27.3 \%)$ & \\
I do not need a Pap test & 0 & $4(100 \%)$ & \\
\hline Note: Patients that answered 'I am not sure how I would feel' (Q4; $\mathrm{n}=13)$ were excluded
\end{tabular}

